\title{
Unfolding the Decision-Making Dynamics of News Visualization Production in China
}

\author{
Qian Ma \\ University of Miami
}

\begin{abstract}
News visualization is a journalistic form that centers on delivering information through data visualizations. It is arousing the interest of journalism professionals and scholars worldwide, including those in non-western societies. Limited studies have identified the challenges and opportunities of news visualization's adoption in non-western newsrooms, but the professionals' considerations and actions to deal with these challenges and seize the opportunities are unknown. This study explores the criteria for a valuable news visualization and how professionals balance the criteria and constraints to make news decisions in the newsrooms in China. A grounded analysis drawing on 15 in-depth interviews with professionals reveals the dynamics of source selection, interpretation, and presentation in news visualization production. It also shows that news visualization stories result from the debating and tradeoffs between the "western norms" and the organization's expectations, audiences' preferences, news commercialization, and macro environment in China.
\end{abstract}

Keywords: Data journalism, news visualization, news decision, news value, China.

Index Items: Human-centered

computing $\sim$ Visualization $\sim$ Visualization application

domains $\sim$ Information visualization

\section{INTRODUCTION}

Data journalism is the journalistic practice that encompasses gathering, analyzing, and interpreting quantitative data in news stories [1]. Over two decades, It emerged and then thrived thanks to the increasing interest in quantitative methods in journalism, the ubiquity of digitized information, big data, and open-source resources (such as tools and learning materials) [2], [3]. Data visualization, the visual representation of data [4], is a powerful data journalism presentation technique [5]. It displays data through charts, graphs, maps, networks, infographics, and motion graphics. It also allows interactive data exploration and analysis such as filtering, aggregating, searching, and scaling [6]. Therefore, it can help both the journalists and the audiences understand massive and abstract information effectively [4][5]. This study uses the term "news visualization" to refer to the branch of data journalism that uses data visualization to convey quantitative information to audiences [6].

News visualization originated in news organizations in Western countries that have a long tradition of applying quantitative methods in news reporting [3], such as the United States (US) and the United Kingdom (UK). Some elite news organizations in these countries have already developed news visualization roles/positions and processes within their graphics teams [7]. These teams can produce numerous news visualization stories with diverse story formats and seek broader cooperation with nonjournalistic partners [8], [9]. In other western countries, such as France, Norway, Sweden, or the Netherlands, news visualization has also been adopted, although more modestly [6], [10], [11].

In recent years, reporting with data visualizations is arousing the

qxm70@miami.edu interest of both journalism professionals and scholars worldwide, including those in non-western societies [14]. However, according to minimal studies, many teams borrow Western data journalism reporting values and processes and try to adapt them to their local media environments, which always bear various pressures and problems in journalists' reporting capability, news sources, organization's financial support, political agendas, and local media environment [12]-[14].

For example, as one of the earliest to adopt news visualization in the non-western world, the prominent Chinese internet media company, Sohu [16], formed the first news visualization team in China [15]. In the following years, many other Chinese news organizations, such as Netease [17] and Pengpai [18], created news visualization production teams and developed stories on a regular basis. However, according to Zhang and Feng (2019), the early adoption of news visualization as a journalism storytelling technique plagued challenges, such as the insufficiency of data resources, lack of quantitative reporting skills, and the burdensome content creation cycle [15].

However, in recent years, news visualization has witnessed a favorable turn driven by the booming data culture in China [12]. Inspired by the worldwide open data movement and increasing significance of big data, governments at all levels in China, have launched open data portals with official data resources on topics like the economy, education, and health [19]. Private organizations, especially giant internet companies such as Baidu [20] and Ali [21], have accumulated massive data, providing vast and rich resources for news visualization reporting.

The increasing data availability broadens professionals' vision, boost their productivity, and enables the broad application of data storytelling in government, market, and other issues with a need to communicate with data, such as political advertisements (the propaganda of the government's strategies and achievements) and clients-based data storytelling products. Besides, Chinese professionals have gained worldwide fame. For example, the news visualization team in Caixin Media won the "GEN 2018 Data Journalism Award for Best Large Data Journalism Team" [22], and the team in Netease got into the shortlist of the 2021 Sigma Awards [23]. Furthermore, during COVID19, Chinese news media and organizations actively use data visualization to deliver information about the pandemic to the public [24],[25], ranging from disease tracking, risk warnings, self-protection guidelines, possible medical treatments, global situations, and special dates like Wuhan's reopening and the Chinese Ching-Ming Memorial Festival. Typical formats include simple charts/graphs, infographics, one-page interactive news stories with data visualizations, news visualization comics, motion graphics/short videos, and news visualization games.

While seeing the emerging opportunities, challenges, and complex relationships within the news visualization field in China, the academic literature of news visualization practice is still minimal. While the existing studies identified the challenges and dilemmas of news visualization's early adoption in China [12] and described the professional works as "dancing with chains" [4], the professionals' considerations and actions to deal with these challenges and seize the opportunities are still unknown. Moreover, how these production strategies are applied in reporting COVID19 also deserves scholar's attention.

In this study, I probe into these problems by investigating how 
professionals make news visualization decisions. Decision-making is a cognitive activity that consists of defining the specific problem, setting up objectives, identifying criteria or constraints, generating alternatives, justifying evidence to select the optimal alternative, developing an execution plan, and finally monitoring the action [27]. In news production, professionals make decisions when generating news topics, gathering and selecting news resources, filtering news-worthy information, determining the story angle and specific details, adjusting the news presentation according to audiences' preference, and choosing a proper way for news diffusion [28]-[30]. And a series of criteria are applied to judge and select story ideas deemed valuable/good enough to be published [31], [32].

Many factors can either benefit or bias professionals' judgment and impact their decision-making in news production [31]. As Shoemaker and Reese (1996) suggest, these factors can be arranged into a hierarchical model from micro to macro: individual, routine, organizational, social institution, and social system [34]. News visualization has many forms, such as the "one-off" project with static charts and graphs [34] and the interactive project that allows readers to explore the real-time dynamic data freely [34]. The hybrid story formats require the journalists and editors to master new techniques to present large-scale complex data [1] [11] and be proficient in visual thinking [6]. Creating such formats also encourages iterative, technology-intensive, multidisciplinary, and collaborative production [6]. As the audiences' news reading preferences going visual and mobile-based, the professionals need to produce more simple, mobile-friendly, and creative forms [12] and tailor their work to social media distribution [26].

Moreover, given the commercialization of news visualization, audiences' features and preferences are maximized and quantified into metrics to evaluate news value [36]. This approach impacts news selection [39] and placement [2] and may induce more commodified news that is soft and traffic-concentrated [39] but less public good and less in-depth reasoning [40]. These factors and trends challenge professionals' experiences, perceptions, and expectations towards the journalistic norms, roles, public good, and truthfulness [41], [42], and consequently influence their news decision-making.

The purpose of this study is to understand how news visualization professionals balance all criteria and constraints to make news decisions. My research questions are: (1) What are the criteria for good/valuable news visualization in China? (2) What are the decision-making dynamics when professionals balance criteria and constraints in news visualization production? My research locates in the Chinese context and examines the adoption of news visualization beyond the Western perspective, contributing to the growing academic interests of data journalism in non-western societies [44], especially the comparison studies of news decision-making across nations [12].

\section{Methods}

This qualitative research seeks to explore the decision-making dynamics and professional considerations in news visualization production in China.

I applied a phenomenological approach to understanding the essence of professionals' journalistic experiences through a series of in-depth interviews [1]. The conversations addressed (1) decisions made in different stages of news visualization production, (2) methods, considerations, interactions, and collaborations in the decision-making process, (3) professionals' perceptions of the values, criteria, and constraints guiding their decisions, and (4) decision-making related to the coverage of COVID19. To comprehensively understand the status quo of news visualization production, I recruited interviewees in different positions [7]. These interviewees should come from different news media and organizations. Moreover, all the interviewees should have started their full-time news visualization job before the pandemic.

A total of 15 news visualization professionals working in six different news media and organizations in China were interviewed over three months from September to November 2020. They were interviewed virtually in Mandarin Chinese via WeChat calls with an average length of 60 minutes. The interview scripts were generated from the recordings, transcribed verbatim, checked, and translated into English. All the interviewees remained anonymous to avoid potential conflict of interest. The interviewees' demographic information is described in Table 1.

Table 1: the interviewees' demographic information

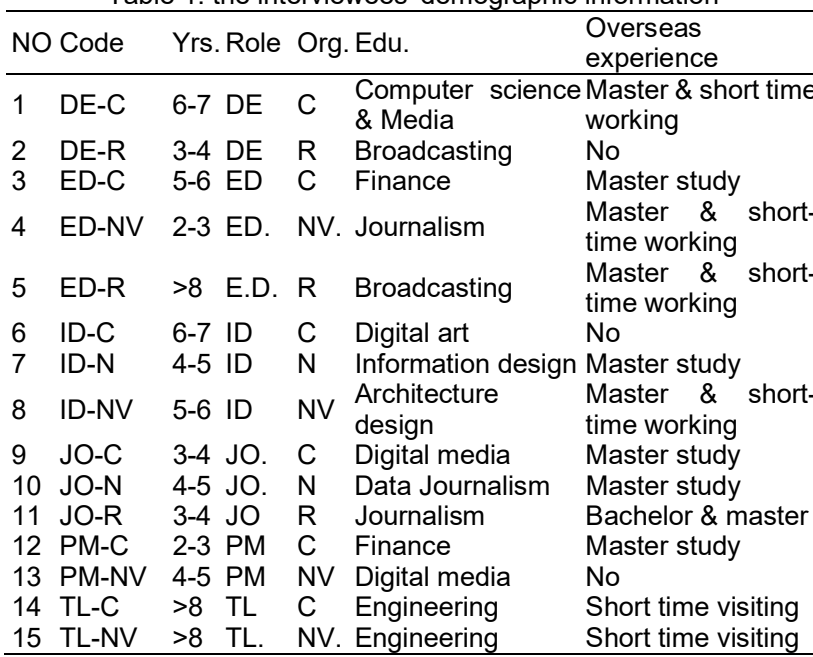

(Note: Yrs. $=$ Years of professional career, Org. $=$ Organization, Edu. =Education; ED=Editor, JO=Journalist, ID=Information designer, $\mathrm{DE}=$ Developer, $\mathrm{PM}=$ Product manager, $\quad \mathrm{TL}=$ Team Leader; $\mathrm{C}=$ commercial media, $\mathrm{NV}=$ News visualization company, $\mathrm{R}=$ Reginal media, $\mathrm{N}=$ National-level media)

Editors (ED) and journalists (JO) are responsible for collecting and processing data, gathering news resources, and writing stories. The two positions are the same in tasks and responsibilities. Information designers (ID) fulfill all design tasks, including infographic layout, interaction design, and webpage design. Developers (DE) provide technical support and produce interactive data visualizations and web pages. Product managers (PM) are responsible for product planning and process monitoring of longterm projects and are involved in story editing and visualization design if needed. Finally, team leaders (TL) control the production team and review products before release.

The news visualization professionals I interviewed belong to four types of media organizations. National-level media $(\mathrm{N})$ refers to the large media outlet that mainly reports national and international news. Regional-level media (R) refers to the organizations that report both local news and national news. Commercial media (C) refers to the private-owned media company that mainly reports news in a specific field, such as education, health, and business. In our sample, this type of team also produces stories about national and international events. Finally, news visualization company (NV) refers to a novel startup company. This type of team produces various data storytelling forms besides news visualizations, such as information dashboards, advertisements, and data art exhibitions.

I applied the grounded theory approach to generate news visualization judgment criteria and explain the decision-making dynamics in news visualization production [45]. I performed systematic coding that consists of open coding, axial coding, and selective coding [45]. Open coding was used to sort and categorize the field data and identify themes constructing the criteria guiding 
the decision-making [46]. The axial and selective coding was used to restructure and cross-compare the data to identify relationships and interactions in the decision-making dynamics [47]. My grounded analysis outcomes were illustrated with two diagrams, the metaphorical visual display [46] of the news visualization judgment framework (Figure 1) and decision-making dynamics (Figure 2).

\section{FINDINGS}

\subsection{RQ1: What are the criteria for good/valuable news visualization in China?}

As shown in figure 1, a visualization story is considered "good/valuable" when it conforms to the "western norms" while being accepted by China's media environment. In detail, a valuable news visualization should meet Chinese audiences' preferences and always be innovative to stand out in a media outlet dominated by conventional news reporting. In some conditions, a news visualization should also have commercial value or value for a political agenda. However, all these values seeking acceptance might be contradictory to "western norms."

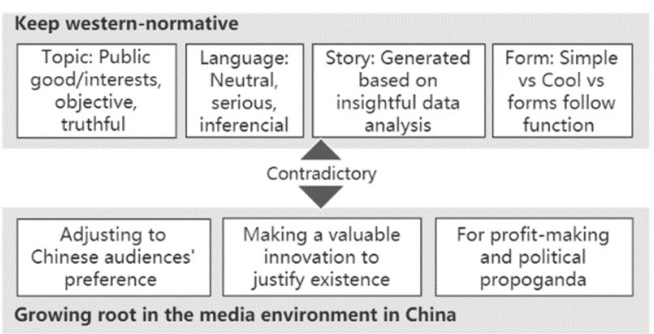

Figure 1, the criteria for good/valuable news visualization in China

\subsubsection{On the Premise of Keeping "Western-Normative."}

News visualization and data journalism were introduced in China by Chinese professionals studying in schools or working for news organizations in the US and UK. These professionals think a valuable news visualization story should be "western normative": addressing public interest, formulated based on truthful and insightful data analysis, and written with objective, neutral, rigorous language.

Journalists/editors and designers/developers express different priorities regarding story formats and visualization forms. For journalists/editors, simple charts, graphs, and maps are ideal for news visualization: "Data visualization is just a tool for delivering information. The value of news visualization stories lies in its story and data analysis" (TL-C, JO-R). They consider those complex data visualizations as showing-off: "These visualizations violate news visualization nature, can be confusing, and even misleading, which are good for nothing except fulfilling designers' desire of creation" (TL-C). But for some developers and designers, a project should be creative and "cool," They mention specific examples of visualization in the western newsroom to illustrate what they mean by "cool." However, others only consider designing "cool visualizations" when joining a data journalism competition. As for regular practice, they hold: "The best form is the one suitable for the certain news topic" (ID-N, ID-NV).

\subsubsection{Growing Root in China's Media Environment}

Most of my interviewees agree that a good/valuable news visualization should help this novel western journalistic form grow deep roots in China.

Adjusting to Chinese audiences' preferences. Reading quantitative news is not a tradition for the Chinese public. "Chinese audiences are expecting the direct news written in one sentence and can be read on their phone, not a long-term story with data reasoning process and complex visualizations" (JO-C, DE-C). Some of my interviewees take audiences' preference as the priority because "A news visualization story is valuable only when it is accepted by the general public, regardless of their genders, ages, social status, and educational backgrounds" (ED-C, JO-N). "A good story is like a bag of street food, not a dish in the Michelin restaurant" (ID-C), "those long-term stories are more suitable for exhibition in the museum" (DE-C, JO-N).

However, some interviewees uphold "western norms" and refuse to tailor their works according to audiences' preferences: "The audiences are a result of two-way selection. Our stories are only for those who like our [western-normative] styles" (ID-NV, TL-C). Furthermore, they express harsh criticism: "The quick news distributed on social media and short video applications, such as Weibo and Tiktok, harm news visualization norms and bring Chinese audiences back to the kindergarten" (ED-R, TL-C).

Making a valuable innovation to justify existence. Producing such "western-normative" stories requires a team of professionals with multidisciplinary skills and mindset, such as data visualization and analysis, interaction design, and frontend/backend development, which is complex, time-consuming, and not costeffective. On most occasions, news media and organizations prefer normalized journalism forms and practices such as broadcasting, textual writing, and photography mainly due to financial considerations. Therefore, news visualization teams struggle against being marginalized or even abolished in their organization. Although all interviewees agree that news visualization has grown in popularity in China due to the massive reporting on the COVID19 pandemic, they still claim they need to justify the value of news visualization to Chinese media organizations and the market.

The only way to justify existence is to keep making valuable innovations. "An innovation means doing something new in either the data sources, topics, analysis, or forms. But it's valuable only when the project is so engaging and stand out that both your peers and audiences are willing to read the whole story and share with others" (JO-N, ED-R). Creating "hot-cake" stories is a quick method for a news visualization team to justify their existence and be irreplaceable in their organization. The hot-cake story refers to super popular projects that occupy massive internet traffic, especially on social media, in a short period. "News visualization field is lacking in hot-cake projects, and that's why we have little financial support and opportunities" (ED-C). However, "the hotcake story relies on luck at the right moment. You may sacrifice news norms and values and totally focus on entertaining your audiences" (JO-R). Consequently, "news visualization will lose its power, narrative with data, and lose the potential to be broadly applied in various scenarios of data communication" (ED-R).

For profit-making and political propaganda. If a news visualization team stays innovative and creates engaging stories, it will become a brand signature for their organization. While the team will receive more financial support and other resources, their organizations will assign them to create client-oriented profitable data stories and advertisements for political propaganda. And according to my interviewees, they get more of such assignments due to news visualization popularity during the COVID19 pandemic.

Professionals' perceptions are diverse across different backgrounds and positions. Journalists and editors claim that there are conflicts between these practices and the nature of news visualization: "Soften the story is inevitable, which means to give up news visualization norms" (JO-C). For designers and developers, these practices are acceptable: "It may limit our desire of creation, but before being a news idealist, we have to make ends meet" (PMNV, ID-NV). The team leaders furtherly address the significance. "This is an inevitable result of commercial transformation in our 
media organization" (TL-C). "If you want to survive, you need a client for financial support and resources, either commercial clients or the government" (TL-NV).

\subsection{RQ2: What are the decision-making dynamics when professionals balance criteria and constraints in news visualization production?}

As shown in Figure 2, the news visualization production consists of story planning and storytelling. Professionals need to decide story angles, storytelling forms, and data collection methods at the story planning stage and select a valuable/good story plan through team-wide discussion. At the storytelling stage, professionals need to appoint the project manager and decide story content and visual layout. Besides, they also need to make quick decisions in reporting breaking news. News visualization criteria and constraints influence their decisions. According to my interviewees, the dynamics are applicable in reporting COVID19.

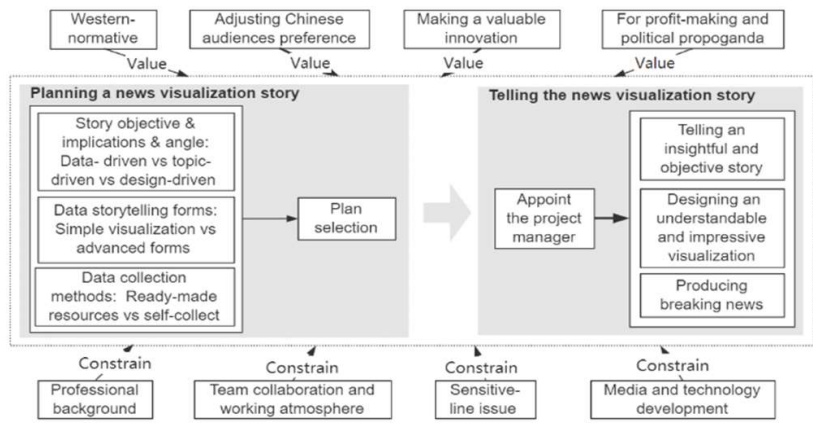

Figure 2, the decision-making dynamics in news visualization production

\subsubsection{Planning a news visualization story}

News visualization story production starts from a tentative plan that includes story objectives and implications, data collection methods, story angles, and storytelling. All team members, regardless of roles and expertise, are invited to propose a story plan.

Generating story objectives, implications, and story angles. The journalists and editors get inspiration by exploring the available data resources (data-driven) while investigating the recent social events (topic-driven). But they need to ensure that the topic can be measured through data analysis and that the findings address public interests. They often seek help from field journalists to generate insightful story angles: "These journalists are experienced in a certain field, such as politics, healthcare, or education, hence would suggest us hidden facts, resources, and advanced analysis methods" (DE-C, JO-N). Designers and developers are more likely inspired by the visualization forms (design-driven). They connect a particular type of visualization with the topic they are interested in (topic-driven) and think about "What information can be presented through this type of visualization (e.g., timeline, map, word cloud)" (ED-C, ID-NV). They assimilate the topic generation as a "mystery": "An interesting story idea is sometimes like a spark in your mind, which relies on years of professional and life experiences" (ID-NV).

Determining the storytelling form. The journalists and editors concentrate on normal storytelling forms such as infographics and long-term stories with simple interactive data visualizations. In contrast, the designers and developers are more active in applying the newest storytelling techniques and create advanced storytelling forms such as data comics, news games, motion graphics, and short videos: "keeping our project forms trendy and evolutive is necessary" (DE-R, ID-C).

To make the plan viable, professionals need to find possible data collection methods. The common ways to collect data sources are referring to the free open database and commercial database. However, this data can be problematic. Either they have incomprehensible variables or notations, or they are unorganized and not updated. And negotiating with private or commercial data providers is often tricky and expensive. The data dilemmas still exist when reporting on COVID-19. Many data providers, especially the local government and Centers for Disease Control (CDC) in rural areas, have limited data disclosure experience and always apply inappropriate data collection and statistical methods. Consequently, the professionals need to cross-check the resources to ensure the truthfulness and accuracy of data.

Given the insufficiency of the ready-made data resources, the professionals always have to collect data themselves, mainly relying on human resources, crawling data from the websites via Python or going into the field, taking notes, photos and videos. However, these methods have pros and cons. The Python crawler is the first choice as it is automatic and flexible. But it requires technical skills and can be invalid when dealing with cryptographic websites. The field data is down-to-earth. But it requires teamwork and good communication skills: "You must be brave enough to take rich photos and videos secretly and have robust techniques to process this messy data" (JO-R). Besides, self-collected data requires attention to privacy, such as personal identity, business, or government secrets. Data collected through personal contacts within large government organizations and big companies can be exclusive. But only national-level media outlets have this type of source within some vital government sectors and giant companies.

Professionals will present their plans at the newsroom meeting. One interviewee assimilates this discussion with "flowing water" (JO-N) because it is non-hierarchical, active, and openminded. "You're welcomed to put forward your own plan and question others while being ready to be questioned" (JO-N). The story should first address the audiences' interests. The selected story plan should be closely relevant to Chinese audiences. "If you want to introduce cultured meat, you should make a comparison between cultured meat and the real meat consumed in China" (JO-R).

Then, the plan should be "western-normative" while making a valuable innovation. The neutrality and objectiveness are important considerations even if the story topic has "hot-cake" potential. For example, a topical phenomenon in China is that students from the bottom of society may not develop a promising career even if they work hard. However, a story visualizing the discussions on social media was killed. Terms like "bottom of society" and "good life career" are neither definable nor measurable, and the participants of the online discussions are unknown. This kind of data analysis and visualization might reinforce the stereotype (students from the bottom).

The professionals keep cautious with those plans applying advanced storytelling techniques. The video format is now popular because "these forms are suitable for mobile and social media distribution and conform to the audiences' preference of quick fragmented reading. We don't need to worry about the loading rate thanks to the high-speed internet traffic in this era of $5 \mathrm{G}$ "(TL-NV, ID-C). However, "These forms might be limited in carrying lots of information and harm the rigorous data reasoning process" (ED-R, JO-N). An interactive story form might engage audiences when the story has a hard-core topic. For example, when reporting the visually impaired groups in China, the team created a first-person perspective through AR (Augmented Reality) on mobile for audiences to empathize with the daily lives of the visually impaired.

In China, controversial or negative topics, such as criticizing the government's strategies and disclosing scandals or corruption cases in some fields, are considered sensitive stories. When a controversial event breaks - for instance, a case of corruption by a government official-journalists know that the government will 
closely monitor their coverage. And journalists know that if they cross any sensitive lines, their stories might be retracted, and they may suffer consequences. But most of the teams won't kill a topic simply for the "sensitive-line" reason: "The definition for the sensitive line is vague and varying all the time. Killing topics blindly is killing workers' motivation and creativity" (ED-R). Instead, they continue working on the topic while informing the chief editor to assess the risk: "They are closer to the government and know the sensitive-lines much better than us" (DE-C, ED-R).

\subsubsection{Telling the news visualization story}

Most of the time, the storytelling process requires a team consisting of a project manager, editor/journalist, designer, and developer. The project manager, the person who first raises the plan, is supposed to deliver his/her own expertise (e.g., design, writing, or developing) while formulating and coordinating the production group, collecting resources, monitoring the process until publication.

Being "western normative" is the priority throughout the whole storytelling process. The sensational title and exaggerated head image will be avoided because "The audiences will feel like being tricked or even cheated if the story content is boring and irrelevant with the title and head image" (ED-R, ED-NV). Moreover, they will turn to field professionals for fact-checking to ensure the story's objectiveness and truthfulness.

The professionals keep news visualization norms tactfully when dealing with sensitive topics, profitable stories, and political advertisements. News visualization is a better way to report sensitive topics because it is developed based on the statistical analysis of many similar events, just like a database, making the single event not obvious or painful. In contrast, conventional news stories often spotlight the single sensitive event "just like a bullet directly hitting the pain point" that will arouse a quick public reaction. However, the news visualization professionals still have to soften the topic to avoid media censorship, though they refuse to brush over the controversial facts. "Being truthful and objective is important, but being publishable is first, or our team will be abolished." For example, when reporting the corrupt officials, they focus on those who have already been convicted instead of those suspicious and under investigation. When reporting the school faculty's sexual assault problems, they extend their scope to all types of abnormal relationships between professors and students. However, some interviewees uphold hard-hitting and critical stories: "We feel the honor to have our story retracted due to the sensitive-line reason." "If our story is insightful and convincing enough, it will be powerful enough to fight with any concealment and push someone to get involved and take on responsibility."

For the profit-making stories and political advertisements, "Although not a news story, they are still data visualization stories delivering information to the public" (ED-C). "It is the producers who decide if the story stands on commercial value or news visualization norms" (JO-C). My interviewees try to develop the story with neutral language and objective analysis, avoiding deliberate exaggeration and flattery. For example, a political advertisement illustrating the achievements of governmental strategies can still be presented based on rational data analysis, providing helpful information to audiences.

Audiences are important. However, instead of totally meeting their preference, the professionals try to help them develop their capacity for reading quantitative reasoning stories. Journalists/editors will embed real-life cases and interviews presented with multimedia forms because these materials are closer to the audiences and more understandable than hard-core data reasoning" (JO-R, JO-N). And the designers will add proper guidance, annotations, or notes to guide their understanding. They will also create good metaphors. For example, when telling the story about a Chinese company's trademark protection action, the designer compared its protection strategies to the moat. Then the infographic was conceived into an ancient battlefield, and the story described a "battle" between the company and the infringing parties. For advanced audiences, such as data scientists, data artists, or quantitative scholars, many media organizations build a crowdsourcing platform for the public to create and publish their own visualization stories. "Their stories may not conform with news visualization norms but can be very insightful because these professionals are much more skilled and knowledgeable than us in data analysis and visualization" (ED-R). The production teams also collaborate with qualified story creators for news visualization story production.

In recent years, new visualization has shown its potential in reporting breaking events, such as natural disasters, traffic accidents, and other emergencies, because "Data visualization is much more comprehensive and intuitive than a verbal paragraph in describing the disaster condition" (DE-C). The team primarily collaborates with the traditional news department because they rely on front-line journalists to collect data resources. The outcome is usually a short-term story with quick data analysis and a simple visualization design. However, news visualization took the lead in reporting the COVID19 outbreak. "Compared to other events, the pandemic is not suitable for on-the-spot reporting because most of the information is abstract and invisible that cannot be collected by the front-line journalists. Thus, the CDC's everyday data disclosure becomes the initial site. Most of my interviewees agree that reporting the breaking events during the COVID-19 brings forth the popularity of news visualization. For example, at the onset of the pandemic, the professionals analyzed 763 cases of virus transmission across the whole country and visualized epidemiological information with network graphs and a timeline. This story became one of the pioneers to reveal the characteristics of COVID19 to the public.

\section{Discussion And Conclusion}

This study explores news visualization judgment criteria, professional considerations, and how, initially a Western journalism form, news visualization is currently being adapted to China's media environment. The dynamics in news visualization production are revealed, such as the story topic and form, data gathering and processing, story development, and publication. The outcome, news visualization story, results from the debating and tradeoffs between the "western norms" and the organization's expectations, audiences' preferences, news commercialization, and macro environment in China.

Professionals' considerations and news judgments are varied according to their background, mindset, and position. The journalists and editors value news visualization norms and keep cautious and critical in applying novel thinking and approaches. They emphasize the public interest/good and are deeply concerned about sacrificing news visualization norms to fulfill audiences' preferences and news commodification. They stay neutral and objective when producing a profitable data story product and tactfully retain the "western normative" when revealing the "sensitive-line" events, even risking retraction. On the other hand, the designers and developers prioritize audiences' needs and preferences and are passionate about applying new forms and technologies. They respect news visualization norms but tend to see the story as a product that values user experience and market acceptance.

The decision-making dynamic is not only built on the team-wide collaboration but also the professionals' interaction with the audiences and clients. The clients can raise needs, reviews, and suggestions, while the audiences can create and publish their own news visualization story via the crowdsourcing platform. At the 
same time, news visualization teams are reaching out and building trust and rapport with traditional news departments. As a result, the hybrid, flexible, collaborative, and multidisciplinary culture bridges the individual differences and alleviates dilemmas like a burdensome creation cycle, insufficient quantitative reporting skills, and an over-rigid hierarchy in China's news organization.

This is a process paper reporting the progress of data journalism production research in China. Further investigations merit two directions: (1) to identify criteria and constraints and understand professionals' tradeoffs in a specific news reporting context, such as coverage of political and economic events, producing mobileand social media-only stories; (2) to compare new visualization judgment framework and decision-making dynamics with teams in other non-western societies.

\section{REFERENCES}

[1] A. Veglis and C. Bratsas, "Reporters in the age of data journalism," journal of applied journalism \& media s, vol. 6, no. 2, Art. no. 2, Jun. 2017, doi: 10.1386/ajms.6.2.225 1

[2] S. C. Lewis and N. Usher, "Open source and journalism: toward new frameworks for imagining news innovation," Media, Culture \& Society, vol. 35, no. 5, Art. no. 5, Jul. 2013, doi: 10.1177/0163443713485494.

[3] M. Coddington, "Clarifying Journalism's Quantitative Turn: A typology for evaluating data journalism, computational journalism, and computerassisted reporting," Digital Journalism, vol. 3, no. 3, Art. no. 3, May 2015, doi: $10.1080 / 21670811.2014 .976400$.

[4] E. R. Tufte, The Visual Display of Quantitative Information, 2nd edition. Cheshire, Conn: Graphics Press, 2001.

[5] A. Figueiras, "Narrative Visualization: A Case Study of How to Incorporate Narrative Elements in Existing Visualizations," in 2014 18th International Conference on Information Visualisation, Paris, France, Jul. 2014, pp. 46-52. doi: 10.1109/IV.2014.79.

[6] G. Smit, Y. de Haan, and L. Buijs, "Visualizing News: Make it work," Digital Journalism, vol. 2, no. 3, Art. no. 3, Jul. 2014, doi: 10.1080/21670811.2014.897847.

[7] A. Cairo, Nerd Journalism: How Data and Digital Technology Transformed News Graphics. Universitat Oberta de Catalunya, 2017.

[8] A. Hermida and M. L. Young, "Finding the Data Unicorn: A hierarchy of hybridity in data and computational journalism," Digital Journalism, vol. 5, no. 2, Art. no. 2, Feb. 2017, doi: 10.1080/21670811.2016.1162663.

[9] M.-F. Santos, A. K. Schapals, and A. Bruns, "Out-of-the-box versus inhouse tools: how are they affecting data journalism in Australia?," Media International Australia, p. 1329878X2096156, Oct. 2020, doi: $10.1177 / 1329878 \times 20961569$.

[10] E. Appelgren and G. Nygren, "Data Journalism in Sweden: Introducing new methods and genres of journalism into 'old' organizations," Digital Journalism, vol. 2, no. 3, Art. no. 3, Jul. 2014, doi: $10.1080 / 21670811.2014 .884344$.

[11] J. Karlsen and E. Stavelin, "Computational Journalism in Norwegian Newsrooms," Journalism Practice, vol. 8, no. 1, Art. no. 1, Jan. 2014, doi: $10.1080 / 17512786.2013 .813190$.

[12] L. Bounegru and D. J. Gray, Eds., The Data Journalism Handbook: Towards A Critical Data Practice. Amsterdam University Press, 2021

[13] N. Fahmy and M. A. majeed Attia, "A Field Study of Arab Data Journalism Practices in the Digital Era," Journalism Practice, pp. 1-22, Jan. 2020, doi: 10.1080/17512786.2019.1709532.

[14] B. Mutsvairo, "Challenges Facing Development of Data Journalism in Non-Western Societies," Digital Journalism, vol. 7, no. 9, Art. no. 9, Oct. 2019, doi: 10.1080/21670811.2019.1691927.

[15] S. Zhang and J. Feng, "A Step Forward?: Exploring the diffusion of data journalism as journalistic innovations in China," Journalism Studies, vol. 20, no. 9, Art. no. 9, Jul. 2019, doi: 10.1080/1461670X.2018.1513814. [16] “数字之道一一深度挖掘事实, 体验资讯可视化的快感-搜狐新闻.” http://news.sohu.com/matrix/ (accessed May 30, 2021).

[17] "数读_网易新闻." https://data.163.com/special/datablog/ (accessed May 30,2021 )

[18] “美数课用数字解读新闻澎湃新闻 -The Paper.” https://www.thepaper.cn/list_25635 (accessed May 30, 2021)

[19] J. A. Gray and L. Bounegru, Data Journalism Handbook 2: Towards a Critical Data Practice. European Journalism Centre, 2019.

[20] “百度数 智平台一百度大数据智能力量.” http://di.baidu.com/?castk=LTE\%3D (accessed May 30, 2021).

[21] "阿里数据." https://dt.alibaba.com/ (accessed May 30, 2021).

[22] "Caixin wins GEN 2018 Data Journalism Award for Best Large Data Journalism Team, a first for a Chinese media outlet - Caixin Global." https://www.caixinglobal.com/2018-06-08/caixin-wins-gen-2018-datajournalism-award-for-best-large-data-journalism-team-a-first-for-a-

chinese-media-outlet-101267017.html (accessed Jun. 15, 2021).

[23] K. S. K. Keng, "Netease DataBlog," The Sigma Awards, Apr. 27, 2021. https://sigmaawards.org/netease-datablog/ (accessed Jun. 15, 2021) [24] J. Qi, "9 Lessons from Chinese Journalists on Covering COVID-19," Global Investigative Journalism Network, Mar. 18, 2020. https://gijn.org/2020/03/18/9-lessons-from-chinese-journalists-on-

covering-covid-19/ (accessed May 30, 2021).

[25] H. Bao, B. Cao, Y. Xiong, and W. Tang, "Digital Media's Role in the COVID-19 Pandemic," JMIR Mhealth Uhealth, vol. 8, no. 9, Sep. 2020, doi $10.2196 / 20156$

[26] D. Baker, Guidebook to Decision-making Methods. Westinghouse Savannah River Company, 2001.

[27] D. Berkowitz and Y. Limor, "Professional Confidence and Situational Ethics: Assessing the Social-Professional Dialectic in Journalistic Ethics Decisions," Journalism \& Mass Communication Quarterly, vol. 80, no. 4, Art. no. 4, Dec. 2003, doi: 10.1177/107769900308000403.

[28] D. Domingo, P. Masip, and I. Costera Meijer, "Tracing Digital News Networks: Towards an integrated framework of the dynamics of news production, circulation and use," Digital Journalism, vol. 3, no. 1, Art. no. 1, Jan. 2015, doi: 10.1080/21670811.2014.927996

[29] S. D. Reese, "Understanding the Global Journalist: a hierarchy-ofinfluences approach," Journalism Studies, vol. 2, no. 2, Art. no. 2, Jan. 2001, doi: 10.1080/14616700118394.

[30] H. M. Kepplinger and S. C. Ehmig, "Predicting news decisions. An empirical test of the two-component theory of news selection," vol. 31, no. 1, Art. no. 1, Apr. 2006, doi: 10.1515/COMMUN.2006.003.

[31] B. Zhong and J. E. Newhagen, "How Journalists Think While They Write: A Transcultural Model of News Decision Making," Journal of Communication, vol. 59, no. 3, Art. no. 3, Sep. 2009, doi: 10.1111/j.14602466.2009.01439.x

[32] P. J. Shoemaker and S. D. Reese, Mediating the Message: Theories of Influences on Mass Media Content. Longman, 1996.

[33] M. Engebretsen, H. Kennedy, and W. Weber, "Data Visualization in Scandinavian Newsrooms," Nordicom Review, vol. 39, no. 2, Art. no. 2, Dec. 2018, doi: 10.2478/nor-2018-0007.

[34] K. Fink and C. W. Anderson, "Data Journalism in the United States: Beyond the 'usual suspects,"' Journalism Studies, vol. 16, no. 4, Art. no. 4, Jul. 2015, doi: 10.1080/1461670X.2014.939852.

[35] J. Maeyer, M. Libert, D. Domingo, F. Heinderyckx, and F. Le Cam, "Waiting for Data Journalism: A qualitative assessment of the anecdotal take-up of data journalism in French-speaking Belgium," Digital Journalism, vol. 3, no. 3, Art. no. 3, May 2015, doi: 10.1080/21670811.2014.976415.

[36] C. Anderson, "Between creative and quantified audiences: Web metrics and changing patterns of newswork in local US newsrooms," Journalism, vol. 12, no. 5, Art. no. 5, Jul. 2011, doi: $10.1177 / 1464884911402451$

[37] R. Zamith, "Quantified Audiences in News Production," Digital Journalism, vol. 6, no. 4, Art. no. 4, Apr. 2018, doi: $10.1080 / 21670811.2018 .1444999$

[38] T. Arenberg and W. Lowrey, "The Impact of Web Metrics on Community News Decisions: A Resource Dependence Perspective," Journalism \& Mass Communication Quarterly, vol. 96, no. 1, Art. no. 1, Mar. 2019, doi: 10.1177/1077699018801318.

[39] P. J. Boczkowski, "The Processes of Adopting Multimedia and Interactivity in Three Online Newsrooms," Journal of Communication, p. 17,2004

[40] J. E. McNealy and L. B. Alexander, "A Framework for Unpublishing Decisions," Digital Journalism, vol. 6, no. 3, Art. no. 3, Mar. 2018, doi: 10.1080/21670811.2017.1301779

[41] S. H. Stocking and N. LaMarca, "How Journalists Describe Their Stories: Hypotheses and Assumptions in Newsmaking," Journalism Quarterly, vol. 67, no. 2, Art. no. 2, Jun. 1990, doi: 10.1177/107769909006700205.

[42] S. Wright and K. Doyle, "The Evolution of Data Journalism: A Case Study of Australia," Journalism Studies, vol. 20, no. 13, Art. no. 13, Oct. 2019, doi: 10.1080/1461670X.2018.1539343.

[43] E. Appelgren and C.-G. Lindén, "Data Journalism as a Service: Digital Native Data Journalism Expertise and Product Development," MaC, vol. 8, no. 2, Art. no. 2, Apr. 2020, doi: 10.17645/mac.v8i2.2757.

[44] B. Mutsvairo, "A New Dawn for the 'Developing' World? Probing Data Journalism in Non-Western Societies," in Data Journalism in the Global South, B. Mutsvairo, S. Bebawi, and E. Borges-Rey, Eds. Cham: Springer International Publishing, 2019, pp. 1-20. doi: 10.1007/978-3-030-2517721.

[45] A. Strauss and J. Corbin, Basics of Qualitative Research: Grounded Theory Procedures and Techniques, Second edition. Newbury Park, Calif.: SAGE Publications, Inc, 1990

[46] C. Y. Tie, M. Birks, and K. Francis, "Grounded theory research: A design framework for novice researchers," SAGE Open Medicine, vol. 7, p. 205031211882292, Jan. 2019, doi: 10.1177/2050312118822927.

[47] J. P. L. Tuazon, J. E. Y. Arcalas, J. M. D. Soliman, and J. M. Opiniano, "Journalists' creative process in newswork: A Grounded theory study from the Philippines," p. 20, 2020. 